\title{
Lagerungstechniken bei Wirbelsäulenverletzungen
}

\author{
Hans-Peter Kaps
}

\section{Zusammenfassung}

Exakte Techniken zur Bergung und Lagerung sowie deren Umsetzung am Unfallort sind wichtige Voraussetzungen um den Verletzten sicher und ohne zusätzlichen Schaden der Klinik zuzuführen. Hebe- und Umlagerungstechniken als auch die Lagerungssysteme sind im Rettungswesen sowie in der Klinik weitestgehend standardisiert. Wesentlich ist ein exaktes Einstudieren der Techniken und die genaue Kenntnis der Funktion der Lagerungssysteme. Der Beitrag befasst sich mit verschiedenen Systemen und Techniken im Rettungswesen und klinischen Bereich.

\section{Einleitung}

Lagerungstechniken bei Wirbelsäulenverletzten mit und ohne neurologischem Defizit sind im Klinikbereich weitestgehend standardisiert. Bergung bzw. Rettung, Lagerungs- und Ruhigstellungsverfahren am Unfallort, insbesondere deren Ausführung werden in den einschlägigen Werken der Unfallchirurgie nur marginal behandelt, in aktuellen Publikationen zum Rettungswesen jedoch eingehender dargestellt. Im Rahmen der Entwicklung der Notfallmedizin stehen heute die unterschiedlichsten Techniken zur Stabilisierung und Bergung bzw. zum Transport von Unfallverletzten zur Verfügung. Wirbelsäulenverletzte sind besonders während der Bergung und dem Transport gefährdet. $20 \%$ aller Unfallpatienten weisen nach einer Analyse der Kassenärztlichen Vereinigung Bayern vom Sommer 2000

OP-JOURNAL 2001; 17: 120-126

(C) Georg Thieme Verlag Stuttgart · New York
[3] eine Wirbelsäulenverletzung auf. Wurde von Geisler et al. [1] in den 60er Jahren noch mitgeteilt, dass von 958 Wirbelsäulenverletzten ohne oder nur mit geringen neurologischen Ausfallerscheinungen am Unfallort bei Einlieferung in die Klinik 29 Patienten eine Lähmung bzw. eine Verschlimmerung derselben eingetreten ist, immerhin 3\%, sehen wir dank moderner Lagerungstechniken lagerungsbedingte Komplikationen eher selten.

\section{Lagerungstechniken}

Im Folgenden werden verschiedene Bergungs- und Lagerungshilfsmittel, sowie -techniken und deren Durchführung am Unfallort, in der Ambulanz, auf der Intensivstation und im allgemeinstationären Bereich dargestellt.

\section{Lagerungs- und Bergungstechniken am Unfallort}

Bergungstechniken und anschließende Lagerung sind in Abhängigkeit von der Unfallsituation, z.B. eingeklemmt im Fahrzeug oder unwegsames Gelände, sowie von der schon am Unfallort evtl. möglichen Höhenlokalisation der Wirbelsäulenverletzung, eng miteinander verknüpft. Prinzipiell sollten Schutzhelme aus Sicherheitsgründen vorsichtig unter Vermeidung von Längszug oder Lageänderung der Halswirbelsäule abgenommen werden. Eine der verschiedenen Techniken ist in Abb.1a-d dargestellt. Es sind grundsätzlich zwei Helfer notwendig. Die einzelnen Handgrifffolgen müssen laut abgesprochen werden.

Bei Bergung und Lagerung des Unfallverletzten sind die einzelnen Handgrifffolgen laut auszusprechen.

\section{$\mathrm{KED}^{\circledR}$-System (Kendrick-Extraction-} Device)

Das auch als Kettenhemd bezeichnete System dient der Rettung und Ruhig- stellung von Wirbelsäulentraumatisierten unter erschwerten räumlichen Bedingungen wie z.B. Bergung aus einem Autowrack (Abb.2a,b). Das System stabilisiert Kopf, Rumpf und Wirbelsäule in Neutralposition, wird im Fahrzeug angelegt, ist röntgendurchlässig und daher erst nach orientierender Diagnostik in der Ambulanz zu entfernen. Für die Immobilisierung der Halswirbelsäule muss ergänzend eine Halskrawatte angelegt werden.

\section{Spine-Board}

Dieses Lagerungsprinzip gehört in Form eines schwimmfähigen Holz- oder Kunststoffbrettes mit Gurtsystem in den USA zum Standard insbesondere zur Wasserrettung (Abb. 3). In der BRD ist es weniger gebräuchlich.

\section{Vakuummatratze}

Die Vakuummatratze ist das mit am häufigsten zum Transport von Wirbelsäulenverletzungen genutzte Hilfsmittel. Gefüllt mit Polysterolkugeln hat sie ein geringes Eigengewicht bei maximaler Stabilität im entlüfteten Zustand. Auch hier sollte zur Stabilisierung einer HWS-Verletzung eine Halskrawatte zusätzlich angelegt werden. Der Verletzte wird zumeist mit der Schaufeltrage oder mittels des Brückenoder des Schaufelgriffes auf der teilentlüfteten und etwas vorgeformten Matratze abgelegt. Bei der Anlage des Vakuums ist auf die Anmodellierung der Lendenlordose und der seitlichen Konturen von Rumpf und Kopf für einen optimalen Halt zu achten. Bei der so genannten „Sandwich-Technik“ wird die Matratze auf den in Bauchlage auf der Schaufeltrage befindlichen Verletzten anmodelliert, evakuiert und anschließend der Patient zwischen Schaufeltrage und Matratze fixiert „en bloc“ auf den Rücken gedreht. Die Matratze ist zur primären Röntgendiagnostik strahlentransparent. 


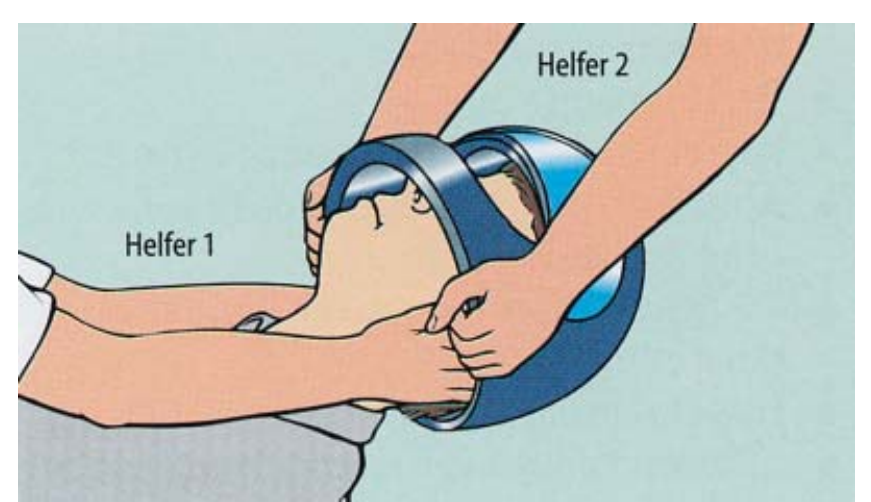

Abb.1a Schutzhelmentfernung

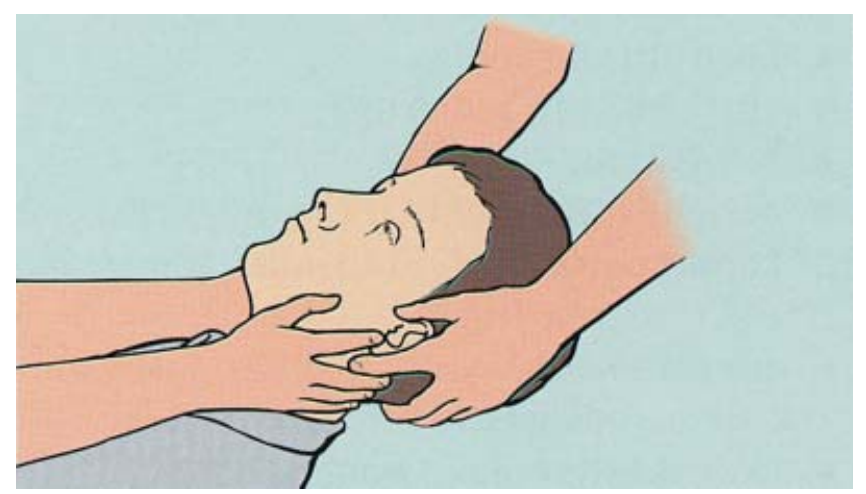

Abb.1c Umgreifen

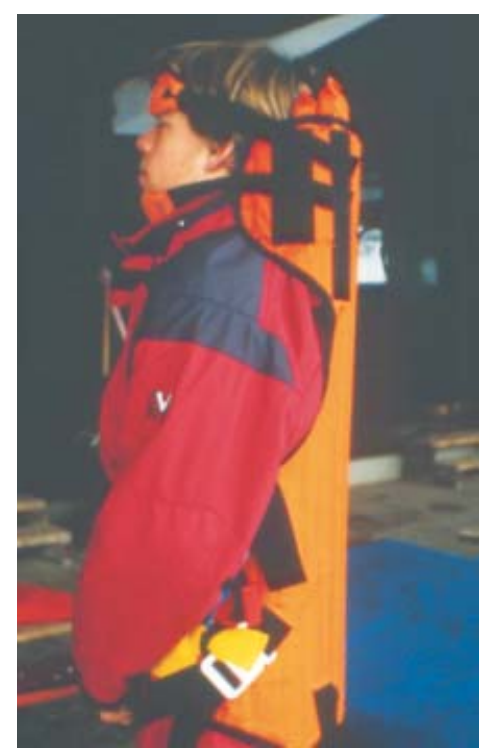

Abb.2a $K^{\circledR}{ }^{\circledR}$-System

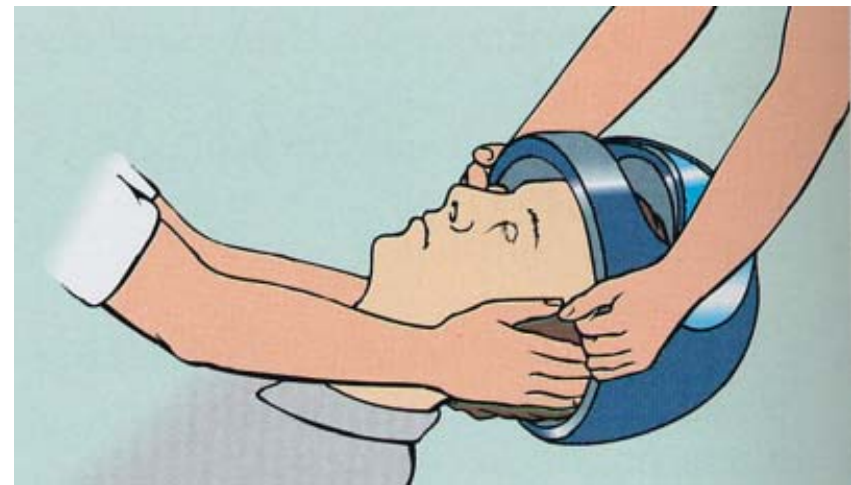

Abb.1b Abziehen ohne Rotation.

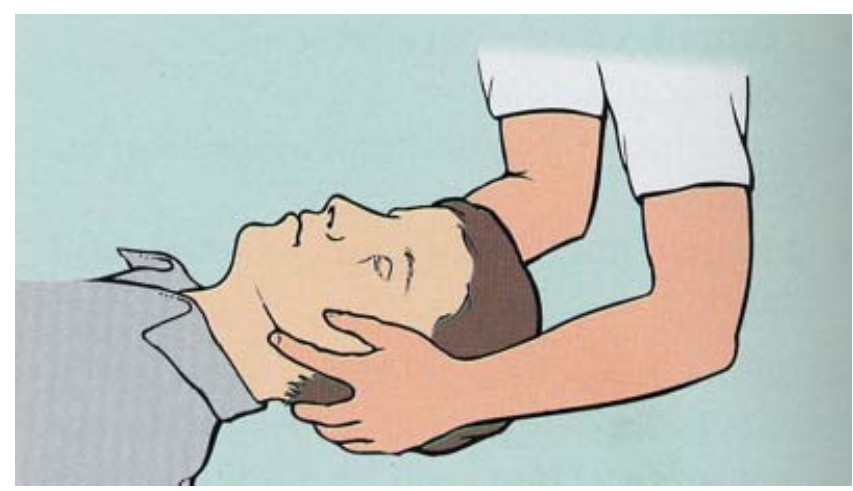

Abb.1 d "In-line“ Stabilisierung (Gorgaß B, Ahnefeld FW, Rossi R, Lippert H-D. Rettungsassistent und Rettungssanitäter. 6. Auflage, Springer, Berlin 2001).
Zur optimalen Stabilisierung des Patienten auf der Vakuummatratze ist die Anmodellierung der Lendenlordose sowie seitlichen Konturen von Rumpf und Kopf unabdingbar.

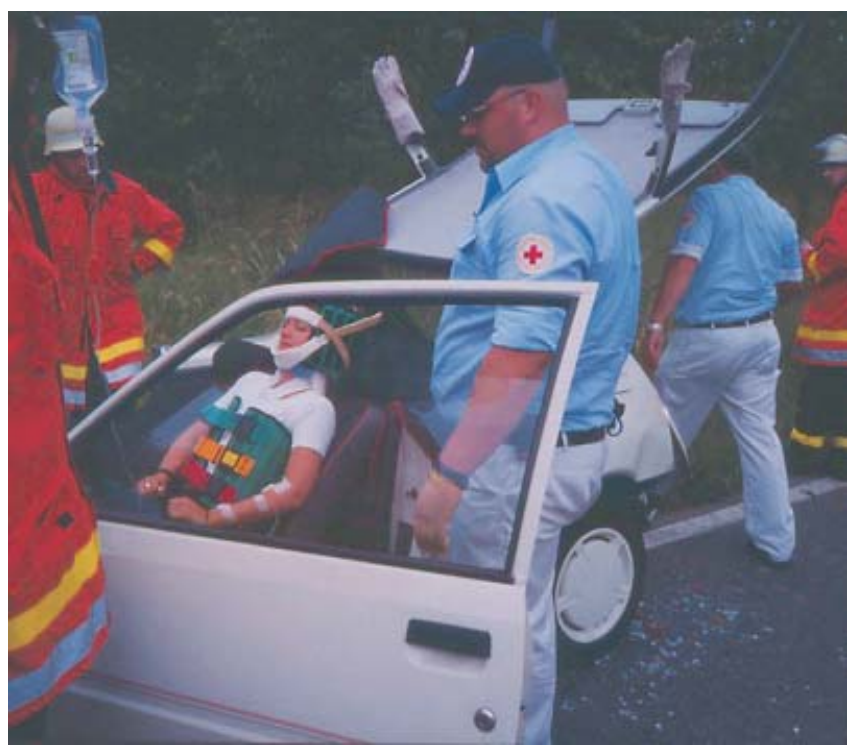

Abb. 2b KED ${ }^{\circledR}$-Anlage im Unfall-PKW (Dr. Simon, Heidelberg).

Halskrawatte

Diese starren Halswirbelsäulen-Fixiermanschetten werden von verschiedenen Herstellern in unterschiedlichsten Aus- führungsformen (Stiffneck ${ }^{\circledR}$, Philadelphia $^{\circledR}$-Halskrawatte, NecLoc ${ }^{\circledR}$ ) ein oder zweiteilig angeboten (Abb.4). Die Anlage erfolgt durch zwei Personen, wobei ein Helfer am Kopfende die Halswirbelsäule 


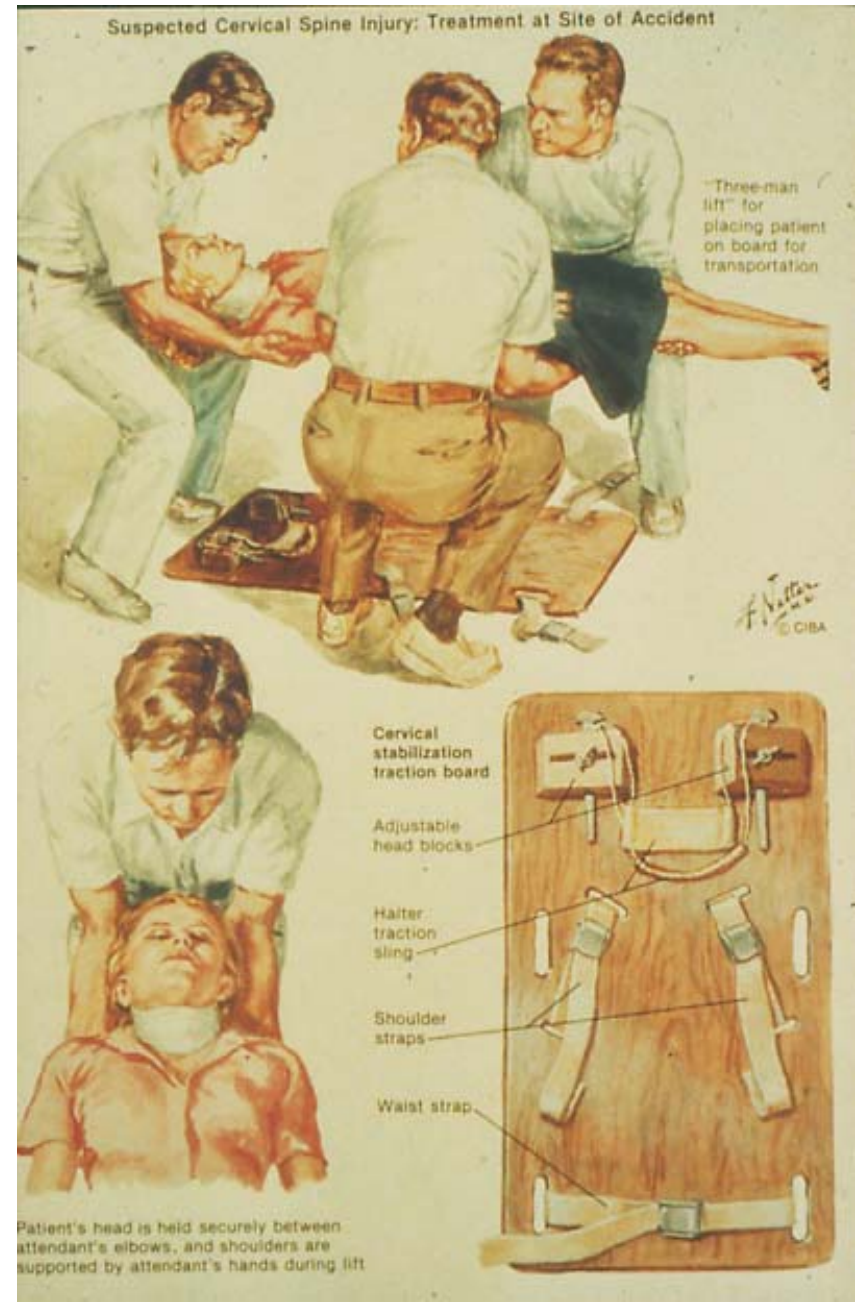

Abb. 3 Spine-Board (Netter-Atlas, The Ciba Collection I - Nervous System).

und den Kopf mittels der manuellen „inline"-Haltetechnik in Neutralposition stabilisiert. Die korrekte Größenwahl ist für eine optimale Immobilisation von Bedeutung. Repositionsmanöver am Unfallort sind obsolet. Lässt sich die Halswirbelsäule nicht zwanglos in Neutralposition bringen, muss sie in der habituell vorgefundenen Stellung stabilisiert werden.

Fehlstellungen der Halswirbelsäule müssen, falls keine zwanglose Einstellung in die Neutralstellung gelingt, in der bestehenden Position fixiert werden. Repositionsmanöver sind obsolet.

Die HWS-Fixiermanschetten reduzieren die Flexionsmöglichkeit um maximal $75 \%$, die der Rotation und Seitneigung um $50 \%$, so dass idealerweise eine zusätzliche Stabilisierung beim Transport auf einer Vakuummatratze erfolgen sollte.

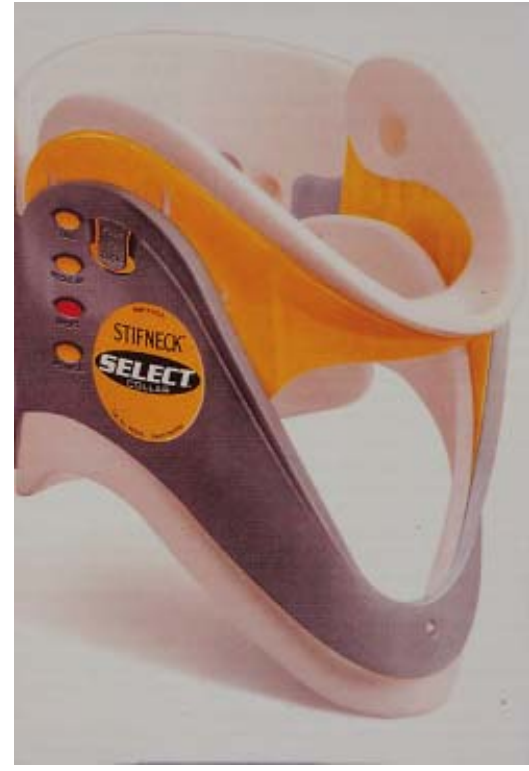

Abb. 4a Stifneck ${ }^{\circledR}$ mit variabler Höheneinstellung (DRK Tübingen).

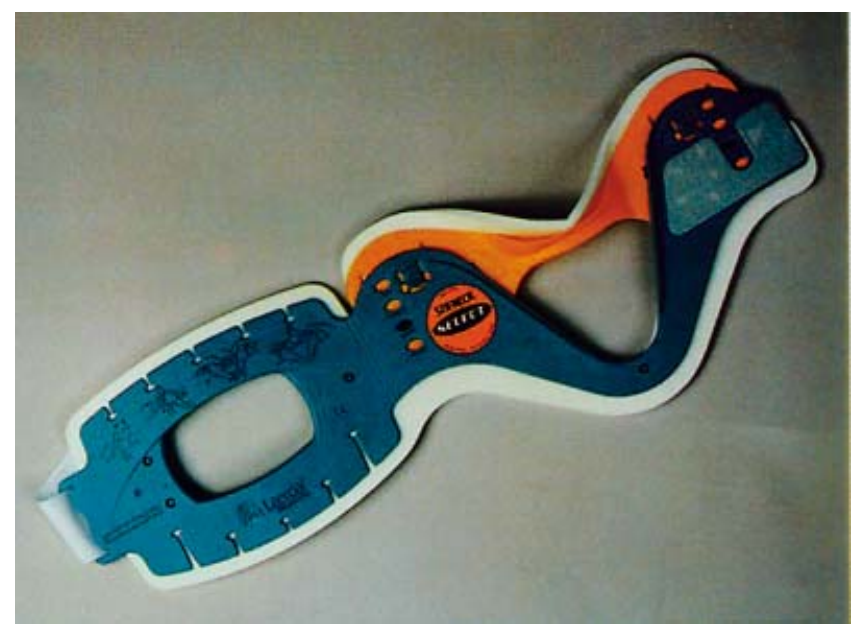

Abb.4b Stifneck ${ }^{\circledR}$ einteilig, geöffnet (DRK Tübingen).

Abb.4c Anlage der zweiteiligen Philadelphia ${ }^{\circledR}$-Krawatte (Gerner HJ. Die Querschnittlähmung: Erstversorgung - Behandlungsstrategie - Rehabilitation. Blackwell Wissenschaft, Berlin, 1992).

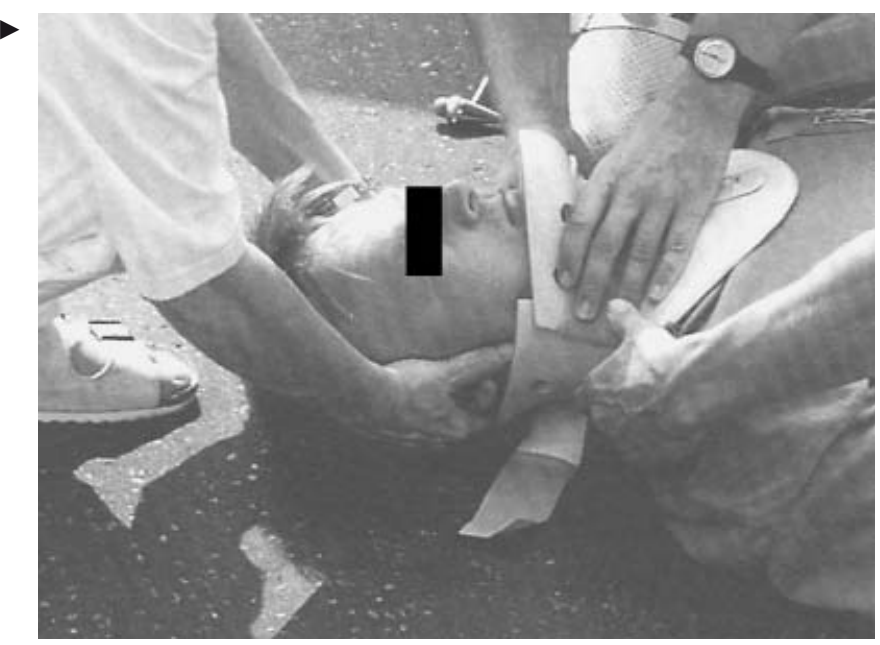




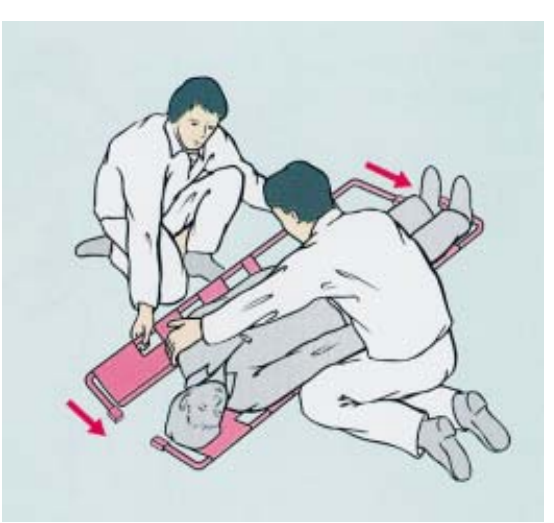

Abb. 5 Anlage der Schaufeltrage

(Gorgaß B, Ahnefeld FW, Rossi R, Lippert H-D. Rettungsassistent und Rettungssanitäter. 6. Auflage, Springer, Berlin 2001).

Sind keinerlei technische Hilfsmittel vorhanden, kann eine Schienung auch behelfsmäßig durch eine entsprechend gefaltete, anmodellierte und mit Pflasterstreifen fixierte Zeitschrift erfolgen.

\section{Schaufeltrage}

Die Schaufeltrage ist unter anderem in Kombination mit der Vakuummatratze ein ideales Gerät zur Umlagerung von Patienten am Unfallort aber auch im ambulanten und stationären Bereich. Sie ersetzt den rückenbelastenden Brückenoder Schaufelgriff. Die Leichtmetallkonstruktion besteht aus zwei der Körpergröße des Patienten anpassbaren Hälften, die an Kopf- und Fußende, nach getrennter seitlicher Unterschiebung unter den Patienten konnektiert werden (Abb.5). Durch leichtes seitliches Anheben ist zu gewährleisten, dass keine Weichteile des Verletzten eingeklemmt werden. Die Schaufeltrage kann auch zur Bergung

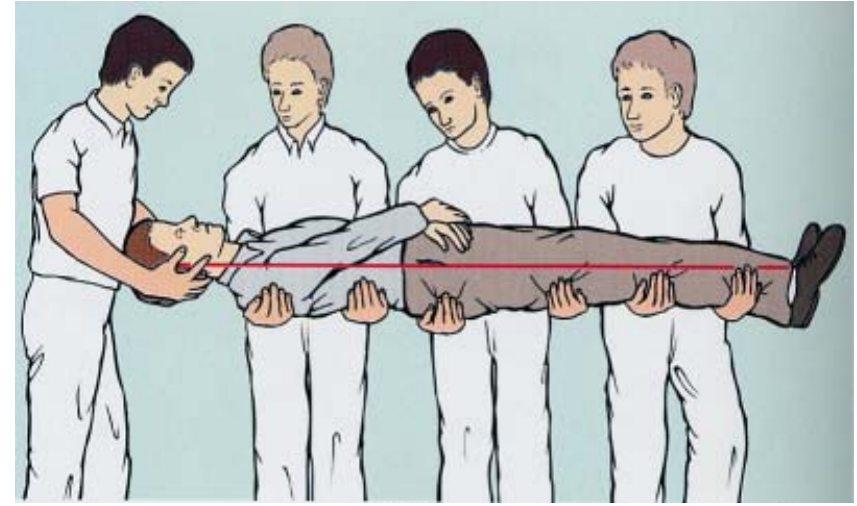

Abb. 6 Schaufelgriff (Gorgaß B, Ahnefeld FW, Rossi R, Lippert H-D. Rettungsassistent und Rettungssanitäter. 6. Auflage, Springer, Berlin 2001).

von Verletzten bei niedrigen räumlichen Verhältnissen eingesetzt werden.

\section{Griff- und Umlagerungstechniken}

\section{Schaufelgriff}

Diese Technik dient der Bergung und Umlagerung des Verletzten z. B. auf eine Vakuummatratze. Sie bedarf mindestens vier Personen (Abb.6). Die Hilfsperson, die den Kopf ablegt, stabilisiert anschließend durch leichtes Anheben mittels der Technik wie beim Brückengriff nacheinander Rumpf, Becken und Beine, so dass der jeweilige „Schaufelgreifer“ seine Arme ohne Bewegung des Verletzten wegziehen kann. Alternativ kann zum Unterschieben als auch Herausziehen der Arme der Patient en bloc um $20^{\circ}$ angedreht werden.

\section{Brückengriff}

Der Brückengriff stellt eine weitere fachgerechte schonende Hebe- und Trage- technik für Lagerung und Ersttransport dar (Abb. 7). Er fordert ebenfalls den Einsatz von mindestens vier Helfern.

\section{Halsschienengriff}

Dieser Griff entspricht der „in-line“-Immobilisierung von Kopf und Hals. Er ist bei der Entfernung eines Schutzhelmes, der Anlage einer Halskrawatte und bei der Umlagerung mittels Brücken- oder Schaufelgriff, wenn noch keine Krawatte angebracht wurde, anzuwenden (Abb. 8).

\section{Lagerung und Umlagerung in der Ambulanz}

In der Ambulanz muss der Unfallverletzte, nach Primärdiagnostik in z.B. der Vakuummatratze am Traumaplatz, zur Durchführung weiterer diagnostischer Maßnahmen wie Computer- oder Kernspintomographie umgelagert werden. Hierzu eignet sich insbesondere die Schaufeltrage oder, wenn diese nicht vorhanden, die Umlagerung mittels mindes-

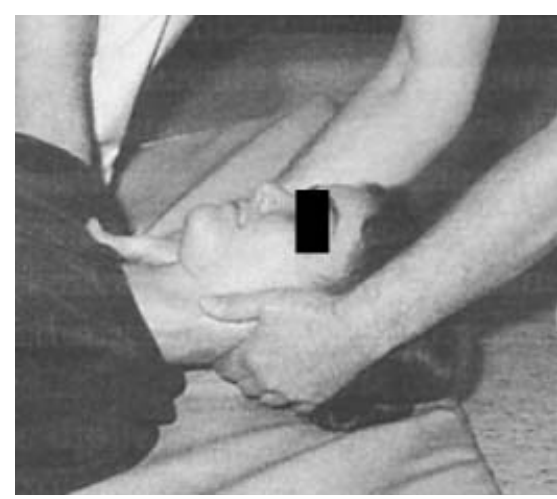

Abb. 8 Halsschienengriff

(Gerner H]. Die Querschnittlähmung: Erstversorgung - Behandlungsstrategie - Rehabilitation. Blackwell Wissenschaft, Berlin, 1992).
Abb. 7 Brückengriff (DRK Tübingen). 


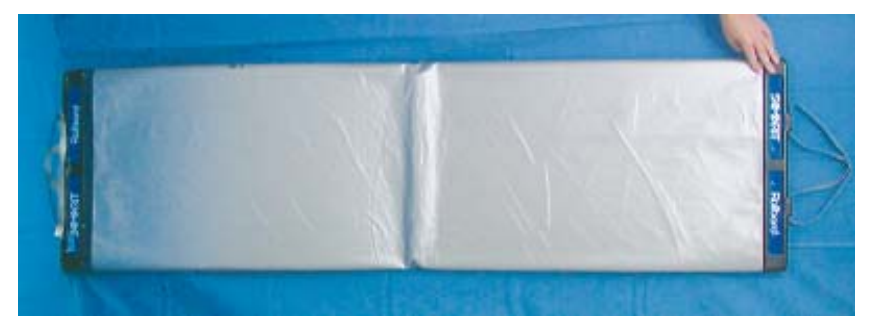

Abb. 9 Rollbord zur schonenden Patientenumlagerung.
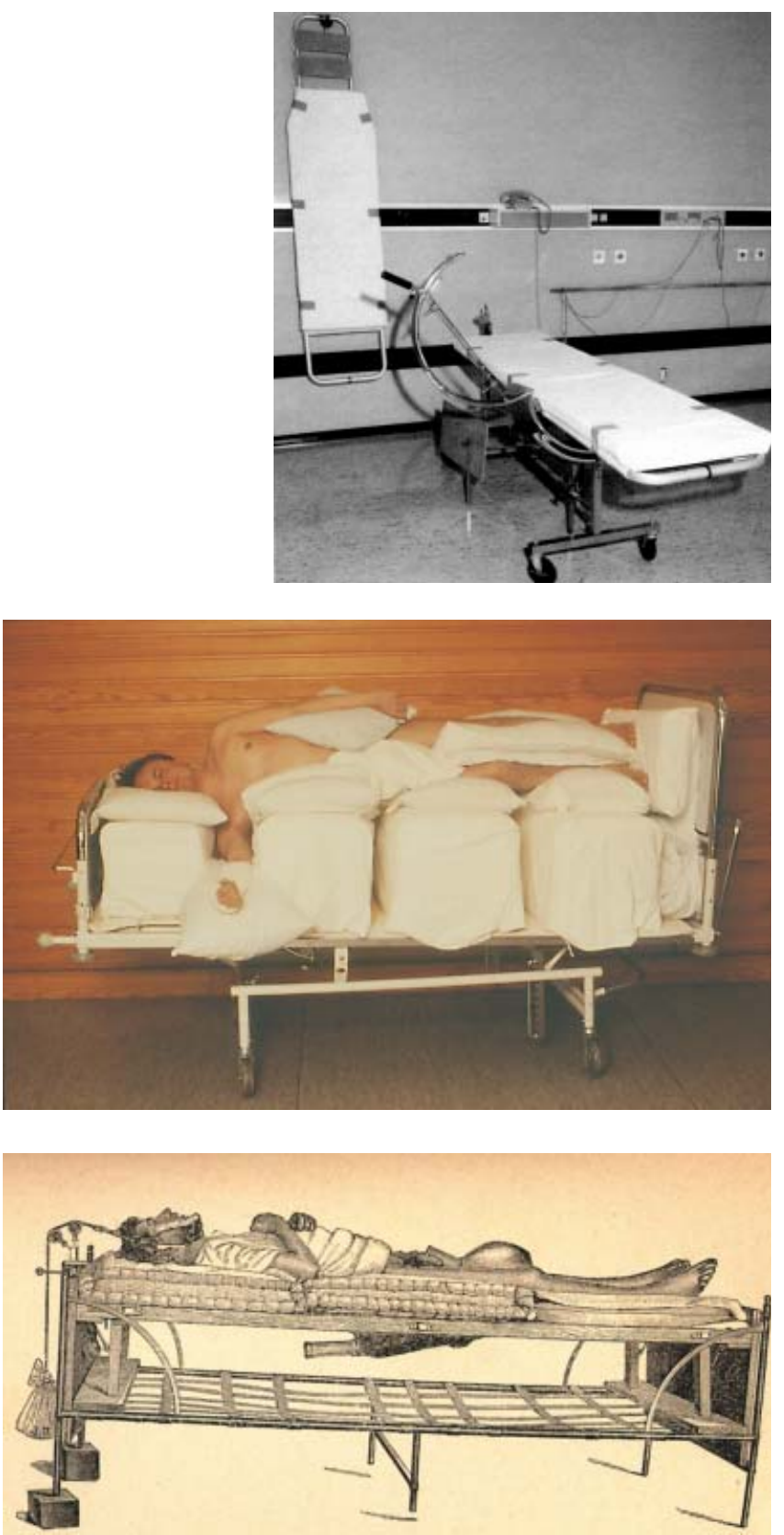

tens vier Personen über den Schaufelgriff. Wird diese letztere Technik bei entkleidetem Patienten angewandt, können zur Erleichterung der Unterfahrung des Patienten mit den Armen, diese im Bereich der Unterarme eingeölt oder eingepudert werden. Alternativ kommt wiederum das vorherige Andrehen infrage. Am ele-
Abb.11 Packbett

Abb.12 Lagerung bei akuter Querschnittlähmung um

1900.

Abb.10 Stryker ${ }^{\circledR}$ -,Sandwich-Bett“ (Gerner HJ. Die Querschnittlähmung: Erstversorgung - Behandlungsstrategie - Rehabilitation. Blackwell Wissenschaft, Berlin, 1992).

gantesten ist jedoch die Anwendung des Rollbordes (Abb.9).

\section{Lagerung und Umlagerung im Operationssaal}

Bezüglich der Umlagerung kommt hier neben der Schaufeltrage das Rollbord oder die Umlagerungsanlage an der OPSchleuse zur Anwendung.

Die Auflage auf dem OP-Tisch sollte fest, jedoch wegen der Dekubitusgefahr nicht hart sein. Gel-Unterlagen sind wegen der nur langsamen Temperaturanpassung ungünstiger als Schaumstoffspezialmatratzen wie z.B. die Tempur $^{\circledR}$-Auflage. Prominente Skelettanteile sind je nach Speziallagerung (dorsaler, seitlicher, ventraler Zugang, Halofixierung) gesondert zu polstern.

Zur Dekubitusprophylaxe sind prominente Skelettanteile gesondert abzupolstern oder hohl zu lagern.

\section{Lagerung auf der Intensivstation}

Je nach Anforderung werden neben Matratzen mit zunehmender Weichlagerung wie Protecto ${ }^{\circledR}$ und Tempur ${ }^{\circledR}$ zur Dekubitusprophylaxe zahlreiche Ausführungen an Spezialbetten auf dem Markt zum Teil auf Leihbasis angeboten. Dies reicht von Luftkissenbetten wie Clinitron ${ }^{\circledR}$ oder $\mathrm{KCl}^{\circledR}$ über Drehbetten wie Stryker $^{\circledR}{ }_{-}$, ,Sandwich-Bett“ (Abb.10), He ${ }^{{ }}{ }_{-}$ Drehbett, Pflegedrehbett Baramed $^{\circledR}$, Schaukelbetten/-Matratzen wie RotoRest ${ }^{\circledR}$ oder KinetiCare ${ }^{\circledR}$, bis zu Wasserbetten wie Hydrofloat ${ }^{\circledR}$. Generell ist zu beachten, dass Spezialmatratzen und -betten die Lagerung nicht ersetzen, der Lagewechsel festgelegt werden muss und sich die Beweglichkeit des Patienten mit zunehmender Weichheit der Matratze verringert. Beim Fluid-Air-Microglaskugelbett weist der Patient aufgrund des Luftstromes einen erhöhten Flüssigkeitsbedarf auf, zudem kann es durch den „Schwebezustand“ zur Orientierungslosigkeit beim Patienten kommen. Entsprechend ist therapeutisch die Technik der „Basalen Stimulation“ notwendig.

Bei längerer Lagerung auf Luftkissenoder Wasserbetten, können die Patienten durch den „Schwebezustand“ unter zunehmender Orientierungslosigkeit leiden.

Lagerung auf der Allgemeinstation/ Querschnittstation

Zur Dekubitusprophylaxe insbesondere für querschnittgelähmte Patienten bietet der Handel verschiedene Modelle mit unterschiedlichen Graden der Druckverteilung und damit Schutzwirkung auf die abhängigen Körperpartien an, wie im Wesentlichen oben unter „Intensivstation" ausgeführt wurde. 


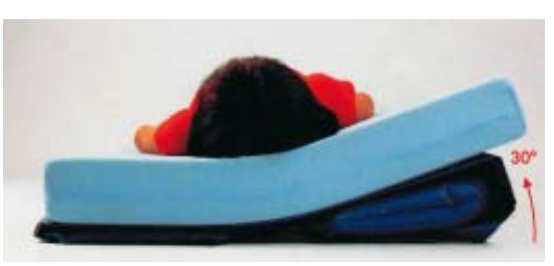

Abb.13 Drehmatratze KinetiCare ${ }^{\circledR}$ (Prospekt Fa. Sacon, Wiesloch).

Das Packbett (Abb.11) wird zur Akutbehandlung bei Querschnittlähmung nur noch selten eingesetzt, hat aber seine Berechtigung bei komplexen Lagerungsanforderungen wie zum Beispiel bei ausgeprägten Kontrakturen der Extremitäten und massiven Fehlstellungen der Wirbelsäule wie beim Morbus Bechterew.

Das Packbett hat nach wie vor seine Berechtigung bei schwierigen Lagerungsvorgaben.

\section{Lagerungsprinzipien bei Querschnittlähmung}

Schon Ende des 19. Jahrhunderts wurden Speziallagerungen für frisch Rückenmarkverletzte publiziert (Abb.12, Helferich 1898), wurden jedoch erst durch Guttmann im Zweiten Weltkrieg in Form des Packbettes konsequent realisiert. Grundsätzlich sind folgende Lagerungsformen bei nicht operativ versorgten Frakturen üblich:

Flache Rückenlage: faltenfreie Unterlage, Fersenfreilagerung und Spitzfußprophylaxe mit je einem Schaumstoffkissen, Knieunterstützung, Nacken mit Rolle unterstützen, spezielle Armlagerung beim
Tetraplegiker. Cave: Druckbelastung von Hinterkopf, Steißbein und Fersen!

Englische Seitenlagerung: Patient wird als Alternative zur flachen RL bei instabilen Frakturen der unteren BWS und LWS „en bloc“ in $30^{\circ}$ Seitlage mit Kissen stabilisiert. Alternativ kommen Spezialmatratzen wie KinetiCare ${ }^{\circledR}$ (Abb. 13) mit automatischer Umlagerung in festlegbaren Zeitintervallen in Frage.

Bauchlagerung: bei unversorgten Frakturen nur mit Spezialbetten wie z. B. dem Stryker $^{{ }^{-}}$, ,Sandwich-Bett“ möglich.

\section{Schlussfolgerung}

Korrekte Bergung, Lagerung und Ruhigstellung sind im Rettungswesen eine erste wesentliche Voraussetzung für die optimale spätere Rehabilitation des wirbelsäulenverletzten Patienten. Entsprechend intensiv müssen Notärzte und Rettungssanitäter in diesen Maßnahmen geschult werden, um frühe Sekundärschäden zu vermeiden. Dies gilt ebenso nach Einlieferung des Patienten für die Mitarbeiter des Krankenhauses bei Lagerungsmaßnahmen am Traumaplatz. Die Lagerung und Umlagerung im stationären Umfeld ist überwiegend dem Verantwortungsbereich der Pflege zugeordnet.

\section{Danksagung}

Dem Leiter der Rettungsleitstelle des DRK Tübingen Herrn W. Bauer und dem ltd. Notarzt im Bezirk Heidelberg Herrn Dr. med. R. Simon sei für die Überlassung von Bildmaterial gedankt.

\section{Literatur}

1 Geisler WO, Wynne-Jones M, Jousse AT. Early management of the patient with trauma to the spinal cord. Med Serv J Can 1966 22: $512-523$

2 Gerner HJ. Die Querschnittlähmung: Erstversorgung - Behandlungsstrategie - Rehabilitation. Blackwell Wissenschaft, Berlin, 1992

3 Gorgaß B, Ahnefeld FW, Rossi R, Lippert H-D. Rettungsassistent und Rettungssanitäter. 6 . Auflage, Springer, Berlin 2001

${ }^{4}$ Helferich H. Lehmann's Medicin. Hand-Atlanten. Bd.VIII, Fracturen und Luxationen. von Lehmann, München, 1898

${ }^{5}$ Lutomsky B, Flake F. Leitfaden Rettungsdienst. 2. Auflage, Urban \& Fischer, München, 2000

6 Schneider Th, Wolcke B, Böhmer R, Merz Th. Taschenatlas Notfall \& Rettungsmedizin. Springer, Berlin, 2000

7 Tscherne H, Blauth M. Tscherne Unfallchirurgie Wirbelsäule. Springer, Berlin, 1998

\section{Prof. Dr. med. Hans-Peter Kaps} Chefarzt

Berufsgenossenschaftliche Unfallklinik Abtlg. f. Querschnittgelähmte, Orthopädie und Rehabilitationsmedizin (AQOR) Schnarrenbergstr. 95 D-72076 Tübingen 